\title{
Stabilization of structure in near-infrared fluorescent proteins by binding of biliverdin chromophore
}

\section{Stepanenko, Olesya V.}

2017-07-15

Stepanenko, O V , Stepanenko, O V , Bublikov, G S, Kuznetsova , I M , Verkhusha, V \& Turoverov , K K 2017 , ' Stabilization of structure in near-infrared fluorescent proteins by binding of biliverdin chromophore ' , Journal of Molecular Structure , vol. 1140 , pp. 22-31 . https://doi.org/10.1016/j.n

http://hdl.handle.net/10138/237057

https://doi.org/10.1016/j.molstruc.2016.10.095

publishedVersion

Downloaded from Helda, University of Helsinki institutional repository.

This is an electronic reprint of the original article.

This reprint may differ from the original in pagination and typographic detail.

Please cite the original version. 


\title{
Stabilization of structure in near-infrared fluorescent proteins by binding of biliverdin chromophore
}

\author{
Olesya V. Stepanenko a, *, Olga V. Stepanenko a, G.S. Bublikov a , I.M. Kuznetsova ${ }^{\text {a }}$ \\ V.V. Verkhusha ${ }^{c, d}$, K.K. Turoverov ${ }^{\text {a, }}$ b \\ a Laboratory of Structural Dynamics, Stability and Folding of Proteins, Institute of Cytology, Russian Academy of Sciences, 4 Tikhoretsky ave., St. Petersburg, \\ 194064, Russian Federation \\ ${ }^{\mathrm{b}}$ Department of Biophysics, Peter the Great St. Petersburg Polytechnic University, Polytechnicheskaya str., 29, St. Petersburg, 194064, Russian Federation \\ c Department of Anatomy and Structural Biology, Albert Einstein College of Medicine, 1300 Morris Park ave., Bronx, NY, 10461, USA \\ d Department of Biochemistry and Developmental Biology, Faculty of Medicine, University of Helsinki, 8 Haartmaninkatu st., Helsinki, 00290, Finland
}

\section{A R T I C L E I N F O}

\section{Article history:}

Received 2 August 2016

Received in revised form

28 October 2016

Accepted 31 October 2016

Available online 2 November 2016

\section{Keywords:}

Bacterial phytochrome

BphP1-FP

iRFP

Near-infrared fluorescent probe

Biliverdin

Stability

\begin{abstract}
A B S T R A C T
Near-infrared fluorescent proteins (NIR FPs) engineered from bacterial phytochromes and their mutants with different location of Cys residues, which able to bind a biliverdin chromophore, or without these Cys residues were studied using intrinsic tryptophan fluorescence, NIR fluorescence and circular dichroism. It was shown that a covalent binding of the biliverdin chromophore to a Cys residue via thioether group substantially stabilizes the spatial structure of NIR FPs. The stability of the protein structure and the chromophore association strength strongly depends on the location of Cys residues and decreases in the following order: a protein with Cys residues in both domains, a protein with Cys in PAS domains, and a protein with Cys in GAF domains. NIR FPs without Cys residues capable to covalently attach biliverdin have the lowest stability, comparable to NIR FP apoforms.
\end{abstract}

() 2016 Elsevier B.V. All rights reserved.

\section{Introduction}

The fluorescence technique gave new opportunities for fundamental research in cell and molecular biology, including monitoring of cellular processes with high-resolution in the real-time $[1,2]$. The engineering and continuous improvement of proteinderived fluorescent markers has greatly contributed to the development of fluorescence microscopy [3,4]. Fluorescent proteins (FPs) developed from bacterial phytochromes (BphPs), which utilize a biliverdin IX $\alpha$ [5] (BV) as a chromophore group, have the most longwavelength absorption and fluorescence spectra among existing fluorescent markers falling into the near-infrared (NIR) spectral region where the biological tissues are the most transparent [6-12].

\footnotetext{
* Corresponding author.

E-mail addresses: lvs@incras.ru (O.V. Stepanenko), sov@incras.ru (O.V. Stepanenko), gsb@incras.ru (G.S. Bublikov), imk@incras.ru (I.M. Kuznetsova), vladislav.verkhusha@einstein.yu.edu (V.V. Verkhusha), kkt@incras.ru (K.K. Turoverov).
}

The strategy to generate NIR FPs from bacterial phytochromes was based on deleting of PHY and effector domains living only PAS and GAF domains, which are minimally required for binding of biliverdin (BV) chromophore [4,7,13]. Further blocking photoconversion and excited-state photon transfer, the competitive excited-state deactivation pathways, was achieved by amino acid substitutions in the chromophore vicinity [11]. Recently it was showed that substitutions of distant from the chromophore amino acids in PAS-GAF of $R p B p h P 2$ photoreceptor from Rhodopseudomonas palustris, may improve fluorescence by favoring the fluorescent state of the chromophore and additionally increasing its quantum yield [14]. It was proposed that these substitutions make the chromophore environment more rigid [14].

The spectral properties of NIR FPs are strictly dependent on the location of Cys residue to which BV chromophore is covalently bound. Red-shifted NIR FPs preserve the conserved for natural BphPs Cys residue (Cys15, here and throughout the text the amino acid numbering is given according to the alignment in Fig. S1) in the $\mathrm{N}$-terminal extension of the PAS domain $[15,16]$. It was shown that in engineered NIR FP, called BphP1-FP, and its variants BV can also bind via a Cys residue introduced into a conservative - SPXH- motif 
of the GAF domain (Cys256), resulting in two BV adducts with different chromophore $\pi$-conjugated systems and $30-35 \mathrm{~nm}$ blue shift of both absorbance and fluorescence [17]. The ability of NIR FPs to bind BV via Cys residue in GAF domain was used for engineering the smallest NIR FPs based only on GAF domain of RpBphP1 photoreceptor from Rhodopseudomonas palustris, termed GAF-FP [18]. The GAF-FP apparently lacks figure-of-eight knot [19], a characteristic feature of bacterial photoreceptor, as knot-forming loop in the GAF domain was deleted during protein evolution to prevent protein aggregation. The figure-of-eight in BphPs was proposed to fix the N-terminal region with Cys15 for chromophore binding, to stabilize the interface between PAS and GAF domains and to tighten the chromophore-binding pocket preventing energy loss during potoconversion [20,21].

In the study of dimeric NIR FPs, called iRFPs, and their mutants with Cys residues in either PAS (Cys15) or GAF domains (Cys256), with Cys residues in both GAF and PAS domains, and lacking the Cys residues it was revealed that BV binding to Cys256 in dimeric NIR FPs is affected by inter-monomer and inter-domain allosteric effects [22]. In dimeric iRFP variants without Cys15, a covalent binding of BV to Cys256 in one monomer allosterically inhibits the covalent binding of BV to another monomer whereas the presence of Cys15 allosterically promotes BV binding to Cys256 in both monomers.

Here we proceed the analysis of covalent and non-covalent binding of BV with NIR FPs on their structure and stability. To this aim we studied the guanidine hydrochloride $(\mathrm{GdnHCl})$-induced unfolding and refolding of mutant variants of four NIR FPs from different sources with Cys residues in either PAS (Cys15) or GAF domains (Cys256), with Cys residues in both GAF and PAS domains, and lacking the Cys residues (Fig. 1). The list of NIR FPs includes
iRFP713 and iRFP682 engineered from Rhodopseudomonas palustris RpBphP2 [9,23], iRFP670 engineered from Rhodopseudomonas palustris RpBphP6 [23] and BphP1-FP developed from phytochrome RpBphP1 from Rhodopseudomonas palustris [17].

\section{Materials and methods}

\subsection{Plasmids, mutagenesis, protein expression and purification}

The BphP1-FP, iRFP713, iRFP670 and iRFP682 genes were amplified and cloned into a $\mathrm{pBAD} / \mathrm{His}-\mathrm{B}$ vector (Invitrogen) using BglII and EcoRI sites. The cysteine mutants of NIR FPs were obtained by site-directed mutagenesis using a QuickChange Mutagenesis Kit (Stratagene).

The NIR FPs and its variants with polyhistidine tags on the Ntermini were expressed in LMG194 host cells (Invitrogen) containing a pWA23h plasmid encoding heme oxygenase under the rhamnose promoter [23]. To initiate protein expression, bacterial cells were grown in RM medium supplemented with ampicillin, kanamycin and $0.02 \%$ rhamnose for $5 \mathrm{~h}$ at $37{ }^{\circ} \mathrm{C}$. Then $0.002 \%$ arabinose was added and cell culture was incubated for additional $12 \mathrm{~h}$ at $37^{\circ} \mathrm{C}$ followed by $24 \mathrm{~h}$ at $18^{\circ} \mathrm{C}$. Proteins were purified using Ni-NTA agarose (Qiagen). The Ni-NTA elution buffer contained no imidazole and $100 \mathrm{mM}$ EDTA. The elution buffer was substituted with PBS buffer using PD-10 desalting columns (GE Healthcare). The final purification was performed with ion-exchange chromatography using a MonoQ column (GE Healthcare). The apoforms of iRFP713/C15S, BphPl-FP/C15S/C256I, iRFP682/C15S/C256I, iRFP670/ C15S/C256I were expressed in LMG194 cells. The overnight LMG194 culture was centrifuged, resuspended in RM medium supplemented with ampicillin, and grown for $2-3 \mathrm{~h}$ at $37^{\circ} \mathrm{C}$; then
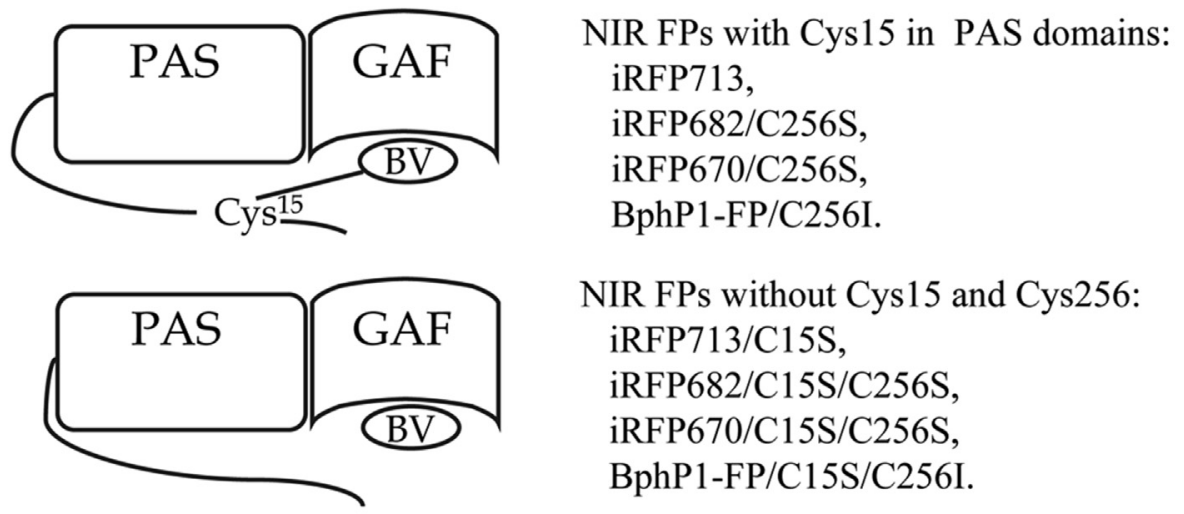

NIR FPs without Cys15 and Cys256:

iRFP713/C15S, iRFP682/C15S/C256S, iRFP670/C15S/C256S, BphP1-FP/C15S/C256I.

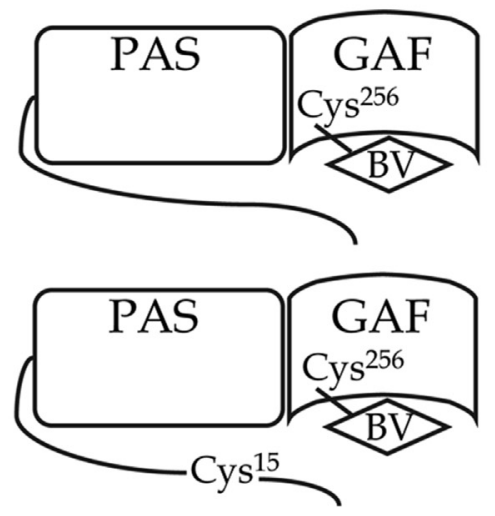

NIR FPs with Cys256 in GAF domains: iRFP713/C15S/V256C, iRFP682/C15S, iRFP670/C15S, BphP1-FP/C15S.

NIR FPs with both Cys15 and Cys256: iRFP713/V256C, iRFP682, iRFP670, BphP1-FP.

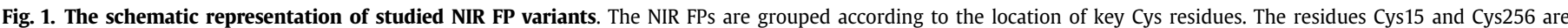

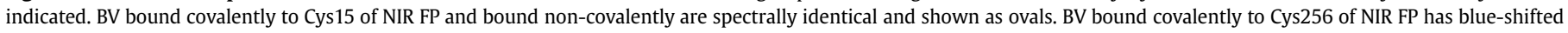
absorption and fluorescence compared to two previous BV adducts and it is shown as diamonds. The variants of NIR FPs belonging to different groups are listed. 
$0.002 \%$ arabinose was added to induce protein synthesis. The subsequent steps of expression and purification of protein in apoform were the same as for proteins in holoform.

The purity of the proteins was analyzed by SDS/PAGE using $12 \%$ polyacrylamide gels [24]. The protein was concentrated and stored in $20 \mathrm{mM}$ Tris/HCl buffer, $\mathrm{pH}$ 8.0. The absorbance of the protein samples did not exceed 0.2 , and the measurements were performed in $20 \mathrm{mM}$ Tris/HCl buffer, $\mathrm{pH} 8.0$, containing $1 \mathrm{mM} \operatorname{tris}(2-$ carboxyethyl)phosphine (TCEP).

\subsection{Spectral characterization of purified proteins}

Absorption spectra were recorded using a U-3900H spectrophotometer (Hitachi). The experiments were performed in 101.016QS microcells ( $5 \times 5 \mathrm{~mm}$, Hellma) with path length of $5 \mathrm{~mm}$ at room temperature. The fluorescence experiments were conducted using a Cary Eclipse spectrofluorometer with FLR cells $(10 \times 10 \times 4 \mathrm{~mm}$; Agilent Technologies, Australia) with path length of $10 \mathrm{~mm}$.

Tryptophan fluorescence in the protein was excited at the longwave absorption spectrum edge $\left(\lambda_{\mathrm{ex}}=297 \mathrm{~nm}\right)$, wherein the tyrosine residue contribution to the bulk protein fluorescence is negligible. The fluorescence spectra position and form were characterized using the parameter $A=I_{320} / I_{365}$, wherein $I_{320}$ and $I_{365}$ are the fluorescence intensities at the emission wavelengths 320 and $365 \mathrm{~nm}$, respectively [25,26]. The values for parameter $A$ and the fluorescence spectrum were corrected for instrument sensitivity. The tryptophan fluorescence anisotropy was calculated using the equation:

$r=\left(I_{V}^{V}-G I_{H}^{V}\right) /\left(I_{V}^{V}+2 G I_{H}^{V}\right)$

wherein $I_{V}^{V}$ and $I_{H}^{V}$ are the vertical and horizontal fluorescence intensity components upon excitement by vertically polarized light. $G$ is the relationship between the fluorescence intensity vertical and horizontal components upon excitement by horizontally polarized light $\left(G=I_{V}^{H} / I_{H}^{H}\right), \lambda_{\mathrm{em}}=365 \mathrm{~nm}[27]$.

\subsection{Analysis of $3 D$ protein structure}

The X-ray data of the CBD (chromophore-binding domain composed of PAS and GAF domains [28]) of bacterial phytochrome RpBphP2 (4E04.ent file) [29] and BphP1-FP/C20S (4XTQ.ent file, the amino acid numbering is given according to the crystallographic data) [17] deposited in the PDB [30] were used as the input data for the analysis of the microenvironment peculiarities of the tryptophan residues localized in the structure of studied NIR FPs. The analysis was performed as previously described [31-34].

The efficiency of non-radiative energy transfer from tryptophan residues to BV chromophore was calculated from the equation [35]:

$W=\frac{1}{1+\frac{2 / 3}{k^{2}}\left(\frac{R}{R_{0}}\right)^{6}}$,

with

$k^{2}=\left(\cos \theta-3 \cos \theta_{A} \cos \theta_{D}\right)^{2}$

where $R_{0}$ is critical Forster distance, at which the probability of energy transfer is 50\%, $R$ is the distance between the geometric centers of Trp indole rings (donors) and BV tetrapyrrole (acceptor), and $k^{2}$ is the factor of mutual orientation of donor and acceptor [36], $\theta$ is the angle between the directions of the emission oscillator of donor and absorption oscillator of acceptor; $\theta_{A}$ and $\theta_{D}$ are the angles between the transition dipoles and the vector connecting the geometric centers of donor and acceptor.

The values of $R$ and $k^{2}$ were evaluated based on crystallographic data for RpBphP2 and BphP1-FP/C20S. The ${ }^{1} \mathrm{~L}_{a}$ oscillator, responsible for the tryptophan emission, was considered to make an angle of $60^{\circ}$ with that of the $\mathrm{C} \delta-\mathrm{C} \gamma$ bond $[37,38]$. The direction of the S4 oscillator responsible for the Soret absorption band of BV (near-UV absorption) was taken according to [39]. The value of $R_{0}$ was determined according to the equation $[35,40]$ :

$R_{0}=9,79 \cdot 10^{3}\left(k^{2} n^{-4} \varphi_{D} J\right)^{1 / 6}$

with

$J=\int_{0}^{\infty} F_{D}(\lambda) \varepsilon_{A}(\lambda) \lambda^{4} d \lambda$

where $\varphi_{D}$ is the donor quantum yield, $n$ is the refraction index of the medium between donor and acceptor, $J$ is a spectral overlap between $\varepsilon_{A}(\lambda)$, the molar extinction coefficient of the acceptor, and $F_{D}(\lambda)$, the normalized to area fluorescence spectrum of the donor. The values of $\varphi_{D}, k^{2}$ and $n$ was accepted to be 0.1 [41], 2/3 (random orientation, [41]) and 1.6 [42], respectively.

\subsection{Equilibrium microdialysis}

Equilibrium microdialysis was performed using a Harvard Apparatus/Amika (Holliston) device, which consists of two chambers ( $500 \mu \mathrm{l}$ each) separated by a membrane (MWCO 10,000) that is impermeable to particles larger than 10,000 Da. Equilibrium microdialysis involves the allocation of two interacting agents, a ligand and its receptor, in two chambers (\#2 and \#1, respectively) divided by a membrane that will allow the ligand to pass but is impermeable to the receptor. In our experiment, NIR FP solution was placed in chamber \#1, and the BV solution was placed in chamber \# 2. The protein and BV were dissolved in $20 \mathrm{mM}$ Tris- $\mathrm{HCl}$ buffer, $\mathrm{pH}$ 8.6, for the microdialysis experiment. The NIR FP concentration was $8-10 \mu \mathrm{M}$, and the BV concentration varied from 10 to $50 \mu \mathrm{M}$. BV hydrochloride (Frontier Scientific) was used without further purification. The BV concentration was determined by an extinction coefficient of $\varepsilon_{376}=45500 \mathrm{M}^{-1} \mathrm{~cm}^{-1}$ [43]. The microdialysis device was left on a rocker for 5 days at $4{ }^{\circ} \mathrm{C}$ to allow the equilibration of the free BV concentration in both chambers [44]. After equilibration, the free $\mathrm{BV}$ concentration $\left(C_{\mathrm{f}}\right)$ in both chambers was equal, while the total BV concentration in chamber \#1 was greater than that in chamber \#2, due to the concentration of the bound chromophore $\left(C_{\mathrm{b}}\right)$, which was determined using the following equation: $C_{b}=C_{0}-2 C_{f}$, where $C_{0}$ is the initial BV concentration in chamber \#2. These data are presented as Scatchard plot and binding parameters determined according to the equation:

$\left(\frac{C_{b}}{C_{p}}\right) / C_{f}=n K_{b}-K_{b} \frac{C_{b}}{C_{p}}$,

wherein $n$ and $K_{b}$ are the number of binding sites and binding constant.

\subsection{Protein unfolding-refolding assay}

The protein unfolding (and refolding) was initiated by mixing $50 \mu \mathrm{l}$ of the native protein (or pre-denatured protein for $8 \mathrm{~h}$ in 4-4.5 M GdnHCl) with $500 \mu \mathrm{l}$ of a buffer solution containing the desired concentration of $\mathrm{GdnHCl}$ (Nacalai Tesque). The 
concentration of the stock $\mathrm{GdnHCl}$ solution was determined by the refraction coefficient. The dependences of the chromophore absorbance, fluorescence, parameter $A$, ellipticity at $222 \mathrm{~nm}$ and light scattering on the $\mathrm{GdnHCl}$ concentration for NIR FPs and its variants were recorded at $23{ }^{\circ} \mathrm{C}$ after protein incubation in a solution of an appropriate denaturant concentration at $23^{\circ} \mathrm{C}$ for $24 \mathrm{~h}$. Further increases in the equilibration time did not result in noticeable changes in the detected characteristics.

The recorded fluorescence intensity was corrected for the total optical density of the solution $\left(D_{\Sigma}\right)$. The corrected fluorescence intensity was defined as $I / W$, where $W=\left(1-10_{\Sigma}^{-}{ }^{D}\right) / D_{\Sigma}$. For details on the correction and normalization, please refer to $[45,46]$. These studies showed that $I / W \cong D q$, where $D$ and $q$ are the absorption and quantum yield of the fluorophore, respectively. Only the corrected fluorescence intensity can be used to evaluate the fraction of molecules that are in different structural states.

\subsection{Circular dichroism measurements}

The circular dichroism (CD) spectra were obtained using a Jasco810 spectropolarimeter (JasCo). The far-UV CD spectra were recorded in a $1 \mathrm{~mm}$ path length cell from 190 to $260 \mathrm{~nm}$, with a step size of $0.1 \mathrm{~nm}$. The near-UV CD spectra were recorded in a $10 \mathrm{~mm}$ path length cell from $320 \mathrm{~nm}$ to $250 \mathrm{~nm}$, with a step size of $0.1 \mathrm{~nm}$. The visible CD spectra were scanned from 800 to $320 \mathrm{~nm}$, with a step size of $0.1 \mathrm{~nm}$, using a $10 \mathrm{~mm}$ path length cell. Three scans were averaged for all of the spectra. The $\mathrm{CD}$ spectra of the buffer solutions were recorded and subtracted from the protein spectra.

\subsection{Fitting of denaturation curves}

The equilibrium dependences of the ellipticity at $222 \mathrm{~nm}$ on the $\mathrm{GdnHCl}$ concentration were fit using a two-state model [47]:

$S=\frac{S_{N}+S_{U} K_{N-U}}{1+K_{N-U}}$

$K_{N-U}=\exp \left(\frac{-\Delta G_{N-U}^{0}+m_{N-U}[D]}{R T}\right)$,

$K_{N-U}=F_{U} / F_{N}=\left(1-F_{N}\right) / F_{N}$,

taking into account

$S_{N}=a_{N}+b_{N}[D]$,

$S_{U}=a_{U}+b_{U}[D]$

where $S$ is the ellipticity at $222 \mathrm{~nm}$ at the measured $\mathrm{GdnHCl}$ concentration; $[D]$ is the guanidine concentration; $m$ is the linear dependence of $\Delta G_{N-U}$ on the denaturant concentration; $\Delta G_{N-U}^{0}$ is the free energy of unfolding at $0 \mathrm{M}$ denaturant; $F_{\mathrm{N}}$ and $F_{\mathrm{U}}$ are the fractions of native and unfolded molecules, respectively; and $S_{N}$ and $S_{U}$ are the signal of the native and unfolded states, respectively; $a_{N}$, $b_{N}, a_{U}$ and $b_{U}$ are constants. Fitting was performed using a nonlinear regression with Sigma Plot.

\section{Results and discussion}

\subsection{Structure of NIR FPs and their variants with different location of Cys residues}

The structural characteristics of NIR FPs in holoforms (i.e. in complex with its BV chromophore) were tested by circular dichroism (Fig. 2) and intrinsic UV-fluorescence (Fig. 3). The intrinsic UV-fluorescence of NIR FPs in apoforms (without BV chromophore) was tested to analyze the contribution of separate Trp residues.

\subsubsection{Structural characteristics of NIR FPs studied by circular dichroism}

The far-UV CD spectra of studied NIR FPs were typical of proteins with mixed $\alpha / \beta$ secondary structure. The far-CD spectra of mutant variants of iRFP713, iRFP682 and iRFP670 exhibited high similarity in the range of $200-250 \mathrm{~nm}$. A slight decrease of $\alpha$-helical content was observed in the NIR FP variants without Cys15 and Cys256 as manifested by less pronounced magnitude of their CD signal in the range of $185-200 \mathrm{~nm}$. Analysis of the far-UV CD spectrum of NIR FPs using the Provencher's algorithm [48] revealed that the content of $\alpha$-helices and $\beta$-sheets was $18 \%$ and $30 \%$ for iRFP713, and $16 \%$ and $29 \%$ for iRFP713/C15S. The far-UV CD spectra of BphP1-FP variants were obviously different from those of iRFP713. The content of $\alpha-$ helices and $\beta$-sheets in BphP1-FP variants was estimated at 13 and $37 \%$. These data disagrees with the results of the X-ray analysis of CBD domain of RpBphP2 [29], the ancestor of iRFP713 and iRFP682, and BphP1-FP/C20S (the amino acid numbering is as in 4XTQ.ent file) [17], showing about $29-30 \%$ and $25 \%$ of $\alpha$-helices and $\beta$-sheets, respectively. The analysis indicated to the diverse secondary structure of BphP1-FP mutant variants compared to the other NIR FPs studied here.

The pronounced CD spectra in the near-UV of NIR FP variants with Cys residues in either PAS or GAF domains, and with both Cys residues indicated at quite rigid and asymmetric environment of aromatic residues in the proteins. The most pronounced near-UV CD spectra observed for NIR FP variants containing both Cys residues, Cys15 and Cys256, probably arise from their more compact structure compared to other studied proteins. The near-UV CD spectra of NIR FPs without residues Cys15 and Cys256 argued for looser packing of side chains of amino acids in these proteins. Thus, the proteins in which biliverdin covalently attached to any of the Cys residues have, apparently, more compact structure. This suggests that a covalent bond between the chromophore and residue Cys 15 or Cys256 is not only mechanically holds the chromophore, but also stabilizes the structure of the NIR FPs.

The studied NIR FPs had typical of bacterial phytochromes in the Pr state the visible CD spectra with a negative band at about 670 or $690 \mathrm{~nm}$ and a positive band at about $390 \mathrm{~nm}$, the position of these bands correspond to the position of the absorption peaks in the visible region.

\subsubsection{Intrinsic fluorescence of NIR FPs in holoforms}

NIR FPs, derived from different bacterial phytochromes, varied in their tryptophan fluorescence spectra markedly (Fig. 3A, Table S1). At the same time, the spectra of tryptophan fluorescence of mutant variants of NIR FP coincided to each other. This suggests that amino acid substitutions did not affect the microenvironment of tryptophan residues of the protein. NIR FPs, engineered on the basis of bacterial phytochrome RpBphP1, RpBphP2 and RpBphP6, have a different number of tryptophan residues: three of them in iRFP713 and iRFP682, Trp109, Trp281, Trp311; two tryptophan residues in iRFP670, Trp95 and Trp281; a single tryptophan residue, Trp281, in BphP1-FP (Fig. S2). The NIR FPs, obtained from different sources, have similar secondary and tertiary structure, as confirmed by X-ray diffraction data. Based on this observation we supposed that the simultaneous analysis of intrinsic UV-fluorescence of these proteins and the peculiarities of the microenvironment of their tryptophan residues allow us to assess the contribution of individual tryptophan residues to the bulk UV-fluorescence of NIR FPs.

The analysis of the microenvironment of the tryptophan 

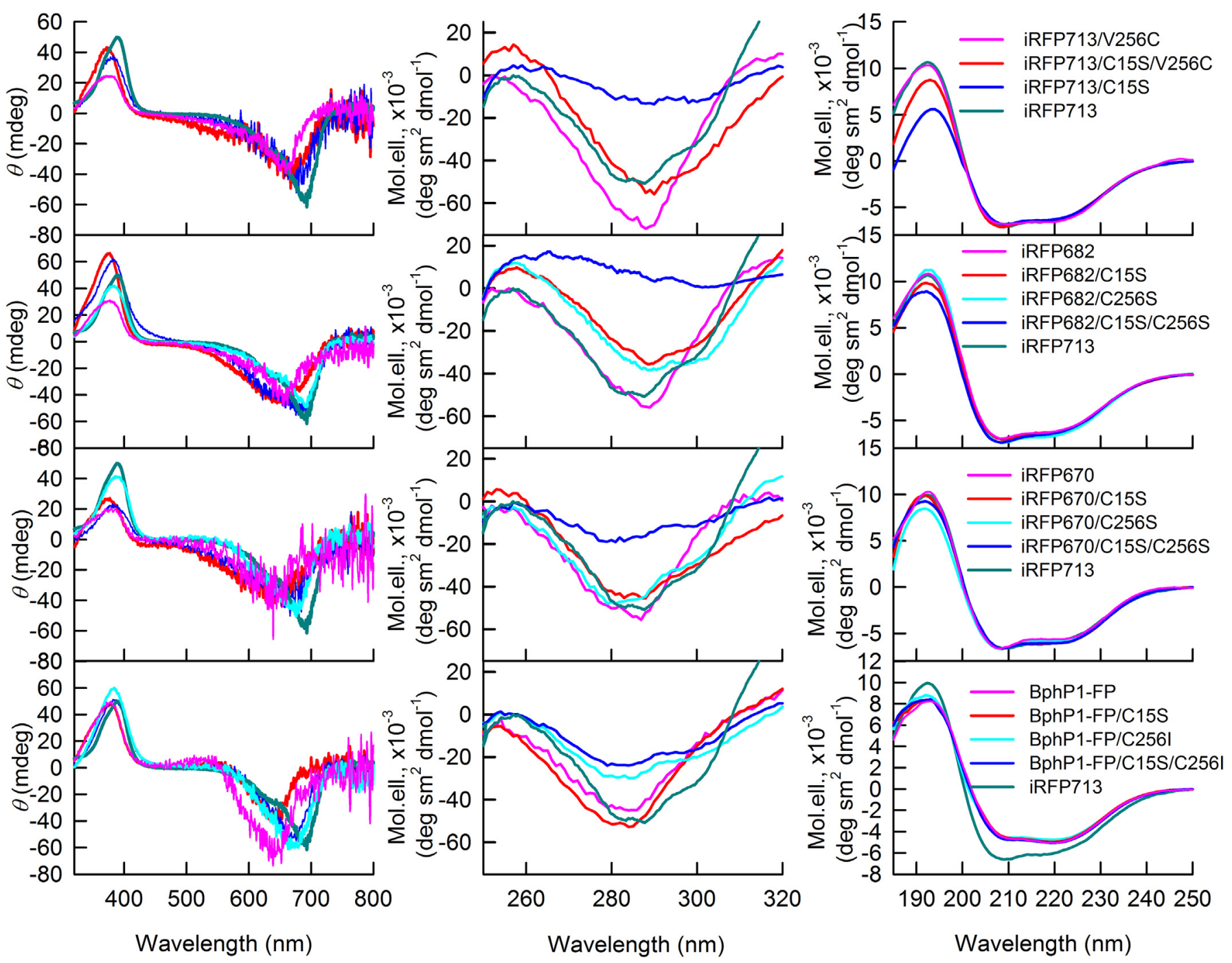

Fig. 2. Characterization of NIR FPs and their variants with different location of Cys residues by CD in the visible, near-UV and, far-UV regions.

residues of iRFP682 and iRFP713 was performed using X-ray data for the RpBphP2-CBD. iRFP713 and iRFP682 differ by three Glu182Val, Thr204Ala, Val256Cys substitutions, none of which is located in microenvironment of Trp residues (according to the $R p B p h P 2-C B D$ structure). Thus we considered the microenvironment of Trp residues in iRFP713 and iRFP682 as similar. The crystallographic data for iRFP670 or it ancestor RpBphP6 is not available. Nevertheless, we can draw some conclusions based on the alignment of the amino acid sequences of iRFP670 with the other proteins.

The analysis of the microenvironment of Trp281, which is present in all NIR FPs, showed its similarity in iRFP713/iRFP682 and BphP1-FP (Fig. S3). Trp281 in iRFP713/iRFP682 and BphP1-FP has rather polar and rigid microenvironment being composed of side chains of Arg174 and Glu317, 5 bound water molecules and rings of Phe175 and Tyr175 (Tables S2 and S3). The microenvironment of Trp281 in iRFP670 is probably less polar with the only polar residue of Arg174 (the number of bound water can not be determined from the sequence alignment) and more rigid with additional two aromatic rings of Phe 313 and His317 except for Phe175 and Tyr173. In the vicinity of $\operatorname{Trp} 281$ there are no groups which are considered to be the most effective quenchers of tryptophan fluorescence such as disulfide bonds [49]. There is SG atom of Cys154 near indole ring of
Trp281 in iRFP713/iRFP682 and SG atom of Cys150 near indole ring of Trp281 in BphP1-FP, but these cysteine residues are not involved in disulfide bonds. The environment of Trp281 in iRFP670 contains none of cysteine residues. Tryptophan fluorescence in proteins can be quenched by electron transfer from indole ring to amid groups of the main chain, this process requires negatively charged groups near the indole ring and/or positively charged groups near amides $[50,51]$. We found no groups able to promote the electron transfer in the microenvironment of Trp281 in iRFP713/iRFP682 and BphP1FP. In contrast, the amide of Trp281 is H-bonded to main chain $\mathrm{O}$ atom of Leu274/Ile274 (2.86 $\AA / 2.84 \AA$ in BphP1-FP/iRFP713), which negative charge may prevent electron transfer (Fig. S3).

The inner tryptophan residue of iRFP713/iRFP682, Trp109, has a very dense and hydrophobic microenvironment, which contains only one molecule of bound water and the single polar residue of Arg111 (Tables S2 and S4, Fig. S3). The bound water molecule is likely H-bonded to NE1 atom of the indole ring of Trp109 being located at a distance of $2.94 \AA$ from it. The presence of electronegative atom near the indole ring of Trp109 may favor the electron transfer. Thus Trp109 in iRFP713/iRFP682 can be quenched. The microenvironment of $\operatorname{Trp} 311$, located at dimeric interface of iRFP713/iRFP682, is formed by side chains of polar amino acids, Gln145 and Gln312, 5 molecules of bound water and side chain of 
A

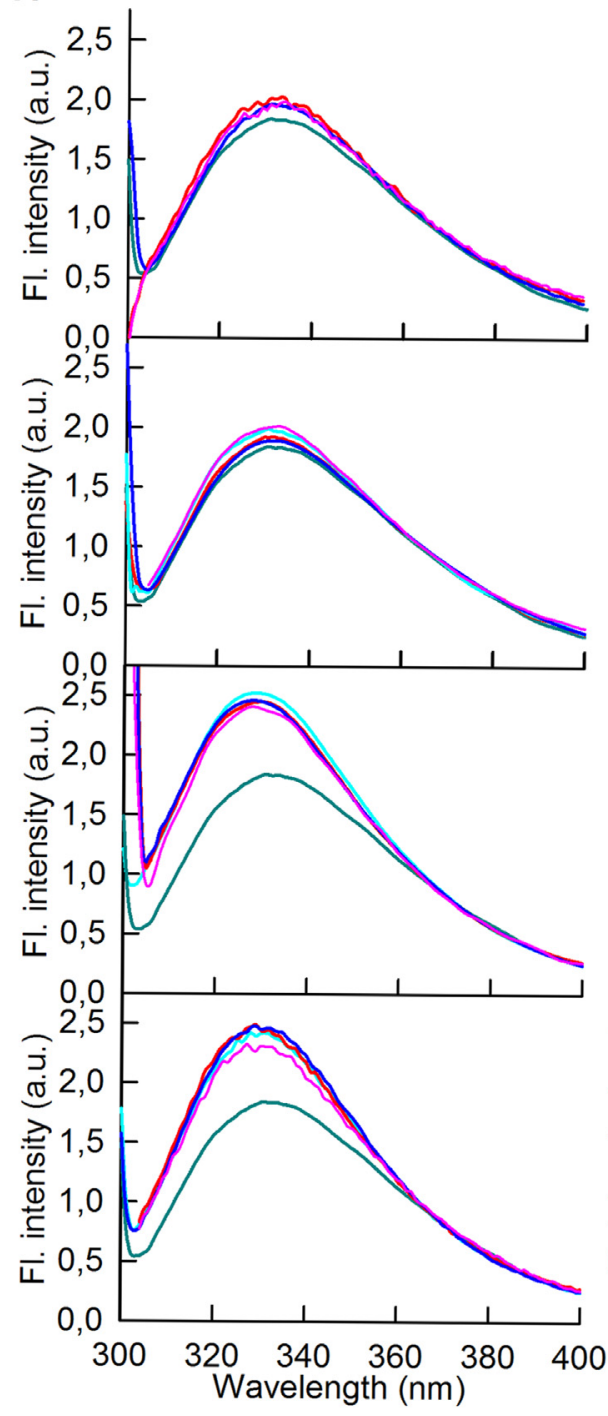

B

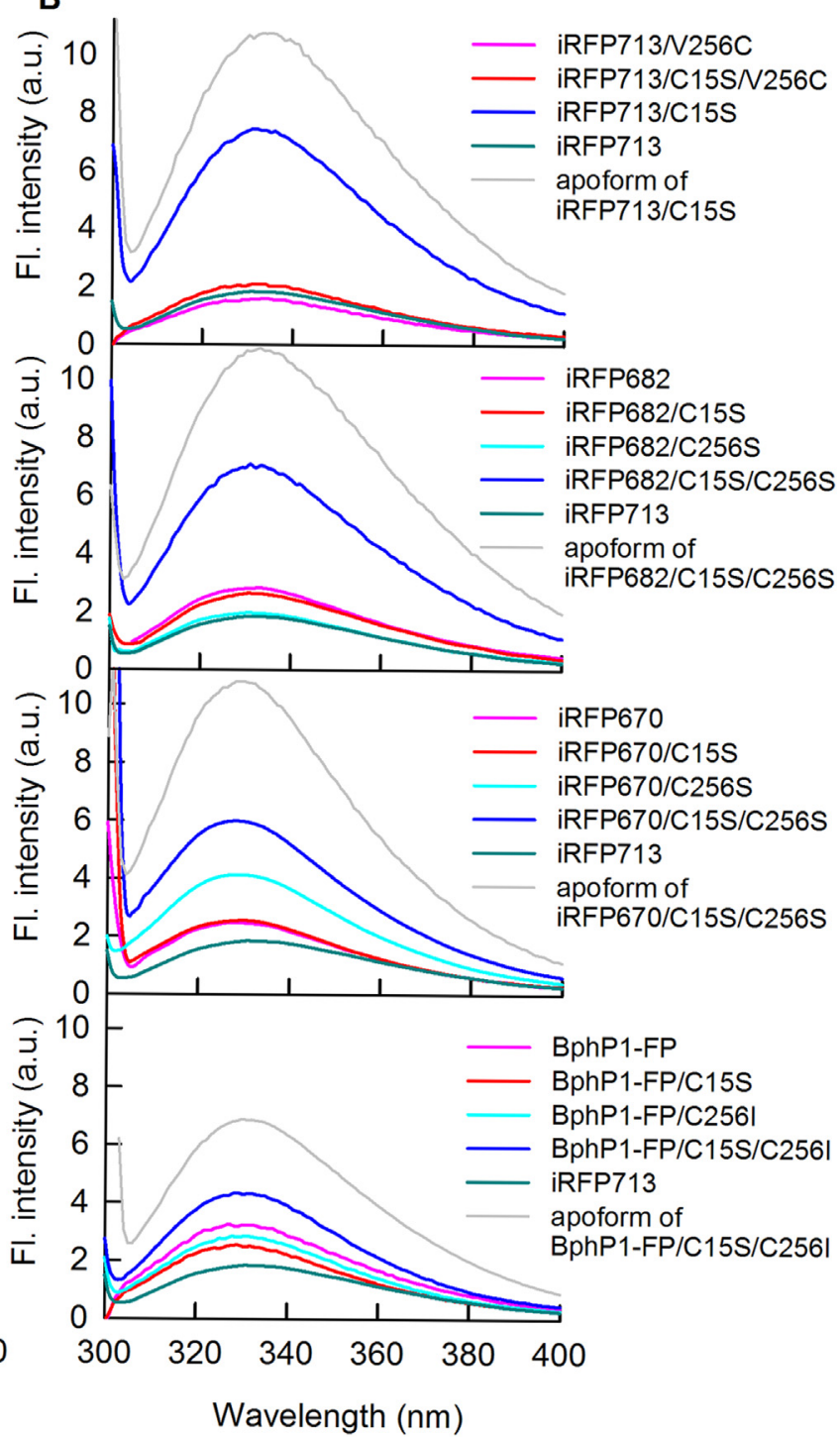

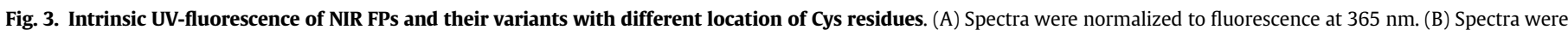
corrected for primary inner filter effect and protein concentration and normalized to the signal of iRFP713 at $365 \mathrm{~nm}$. Fluorescence was excited at $295 \mathrm{~nm}$.

Trp311 of the other monomer (Tables S2 and S4, Fig. S3). No effective quenchers of fluorescence are present near indole ring of Trp311 (Tables S2 and S4, Fig. S3). The peculiarities of the microenvironment of Trp95 in iRFP670 can not be analyzed.

The tryptophan fluorescence intensity of NIR FPs without Cys15 and Cys256 was much larger than that of NIR FPs, with Cys residues in either PAS or GAF domains, or with both Cys residues, but less than the intensity of intrinsic fluorescence of NIR FPs in apoforms (Fig. 3B). Tryptophan fluorescence in the NIR FPs can be quenched by efficient transfer of excitation energy from tryptophan residues to BV. Indeed, the emission spectrum of tryptophan residues significantly overlaps with Soret band in the absorption spectrum of BV (Fig. S4). Previously, it has been shown that tryptophan residues distant from the heme up to $20 \AA$ in heme-containing myoglobins are effectively quenched by prosthetic group [52]. Our calculations of $R_{0}$ gave the value of about $25.5 \AA$. Based on the relative distances between the geometric centers of Trp indole rings and $\mathrm{BV}$ tetrapyrrole and their mutual orientation the efficient fluorescence quenching can be expected in the case of Trp109 and Trp281 (Fig. S2 and Table S5).
In NIR FPs without Cys15 and Cys256 the BV incorporation is reversible. Thus, the solution of NIR FPs without Cys15 and Cys256 contains protein/BV complex and apoprotein which portions are determined by the value of dissociation constant. In the experiments on equilibrium microdialysis the binding parameters of BV with apoforms of NIR FPs were found to be of micromolar range (Fig. S5). The portion of holoprotein in NIR FPs obtained in cell medium was estimated to be not less than $96 \%$ taking the molar ratio of $\mathrm{BV}$ to apoprotein of 10:1 according to the condition of protein expression. The molar ratio of $\mathrm{BV}$ to apoprotein of $1: 1$ gives the portion of holoprotein from 70 to $90 \%$. The presence of the apoprotein impurities in NIR FPs extracted from cells may increase the quantum yield of NIR FPs (Fig. 3B). On the other hand, the looser structure of NIR FPs without residues Cys15 and Cys256 and, hence, increased distances between the Trp residues and noncovalently bound chromophore may result in less effective quenching of the intrinsic fluorescence compared to those proteins with Cys residues in either PAS or GAF domains, or with both Cys residues. This suggestion is in agreement with near-UV CD data. Apparently, BV covalent binding to a cysteine residue stabilizes the structure of NIR FP. 


\subsubsection{Intrinsic fluorescence of NIR FPs in apoforms}

We compared the spectra of tryptophan fluorescence of the NIR FPs in apoforms, where the intrinsic fluorescence is not quenched by the chromophore (Fig. S6, Table S1). It was revealed that the value of intrinsic fluorescence for apoform of iRFP713/C15S, iRFP682/S15S/C256S and iRFP670/S15S/C256S was similar and twice as much as that for apoform of BphP1-FP/S15S/C256I. Based on this, we assumed that bulk UV-fluorescence is determined by Trp281 and Trp311 in iRFP713/iRFP682 variants, while Trp108 is quenched (Table 1). Both Trp281 and Trp95 of iRFP670 and the single Trp281 of BphP1-FP contribute to the protein fluorescence. All these tryptophan residues, probably including Trp95, have rigid microenvironment, which results in blue-shifted tryptophan fluorescence, in spite of polar groups in the microenvironment of the tryptophan residues [32,53]. The most blue-shifted fluorescence of iRFP670 mutant variants among all NIR FPs can be explained by the highest hydrophobicity of the microenvironment of $\operatorname{Trp} 281$ in iRFP670. Slightly red-shifted fluorescence of iRFP713/iRFP682 compared to BphP1-FP can arise from the environment of Trp311 as Trp281 has identical microenvironment in the proteins.

\subsection{The unfolding-refolding processes of NIR FPs and their variants with different location of Cys residues}

Previously we showed that the renaturation of iRFP713 was complicated by the accumulation of partially folded state of protein and its aggregation [44]. The irreversibility of iRFP713 denaturation was associated with the covalent binding of BV chromophore. The figure-of-eight knot was not interfere with protein refolding [44]. We hypothesized that the threading of $\mathrm{N}$-terminal region through the knot-forming loop of GAF domain may be complicated by BV covalently bound to Cys15 thus preventing iRFP713 from correct refolding. To determine how BV localization in the protein structure might affect reversibility of protein unfolding, we studied the denaturation-renaturation processes induced by $\mathrm{GdnHCl}$ of NIR FPs with different localization of Cys residues capable of covalent binding of the chromophore and NIR FPs without these Cys residues. Fig. 4 summarizes quasi-equilibrium curves of parameter $A$, chromophore fluorescence recorded at maximum of visible fluorescence, and the ellipticity at $222 \mathrm{~nm}$ as a function of $\mathrm{GdnHCl}$ concentration for iRFP682/C15S, iRFP682/C256S and iRFP682/C15S/ C256S. Data corresponding to the $\mathrm{GdnHCl}$-induced unfolding and refolding of iRFP670/C15S, iRFP670/C256S and iRFP670/C15S/ C256S are shown in Figs. S7-S9. Figs. S10-S12 contain data for BphP1-FP/C15S, BphP1-FP/C256S and BphP1-FP/C15S/C256I.

We did not observe the recovery of recorded characteristics at renaturation of NIR FPs with covalently attached BV via Cys 15 or Cys256. Similarly to iRFP713, the renaturation of NIR FPs with covalently attached BV was accompanied by protein aggregation as tested by the increase of light scattering at denaturant concentration less than 1.0M for mutant variants of iRFP682 and iRFP670, and less than $1.5 \mathrm{M}$ for mutant variants of BphP1-FP. Current data demonstrated that the change of BV attachment from PAS domain to GAF domain did not improve the refolding of BphP-derived NIR FPs. GdnHCl-induced unfolding of iRFP682/C15S/C256S and iRFP670/C15S/C256S, which do not contain Cys15 and Cys256 and are not able to covalent attachment of the chromophore, was reversible. The structural parameters (ellipticity at $222 \mathrm{~nm}$ and parameter $A$ ) and chromophore characteristics (visible absorption and fluorescence) of iRFP682/C15S/C256S and iRFP670/C15S/C256S measured at the refolding experiment at decreasing of $\mathrm{GdnHCl}$ concentration achieved those of native protein transferred in the same $\mathrm{GdnHCl}$ concentration. These data proved the recovery of protein structure including the chromophore-binding pocket in GAF domain. Thus the covalently bound chromophore hindered the refolding of NIR FPs, probably by emerging of non-native contacts of $\mathrm{BV}$ with protein.

In contrast to iRFP682/C15S/C256S and iRFP670/C15S/C256S, unfolding of BphP1-FP/C15S/C256I was irreversible and complicated by pronounced aggregation of protein molecules. We suppose that BphP1-FP/C15S/C256I is monomeric as BphP-FP/S20S was crystallized in monomeric form according to X-ray data [17]. Probably, inter-monomer contacts in dimeric iRFP682/C15S/C256S and iRFP670/C15S/C256S can stabilize the proteins in native state during refolding while inter-molecular contacts in monomeric BphP1-FP/C15S/C256I can stimulate protein aggregation and inhibit its renaturation.

\subsection{The stability of NIR FPs and their variants with different location of Cys residues}

The effect of the localization of cysteine residues in PAS and GAF domains on the stability and the chromophore binding of studied proteins was analyzed (Fig. 5). NIR FPs were ranged according to the decrease in the stability of their structure and the strength of the chromophore binding as following: NIR FPs with Cys15 and Cys256 > NIR FPs with Cys15 in PAS domains > NIR FPs with Cys256 in GAF domains > NIR FPs without Cys15 and Cys256 $\approx$ NIR FPs in apoform.

Our previous studies of dimeric NIR FPs revealed that the interaction of BV with these proteins was highly affected by intermonomer and interdomain allosterical effects [22]. Allosterical influence of one monomer to the other in dimeric NIR FPs, with Cys residues in GAF domains only, leads to the covalent binding of BV with Cys256 in one monomer and non-covalent binding of BV in other monomer. The suppression of covalent binding of $\mathrm{BV}$ in the second monomer was observed for iRFP682/C256S also [22]. The presence of non-covalently bound BV in iRFP682/S256S is implicit for protein in buffered solution, but it makes obvious after addition to the protein even small amount of $\mathrm{GdnHCl}$. Sequential dissociation of non-covalently bound chromophore at small $\mathrm{GdnHCl}$ concentrations and covalently bound to Cys15 chromophore at modest $\mathrm{GdnHCl}$ concentrations is reflected at the dependences of fluorescence and absorbance, measured at the maximum of visible

Table 1

The contribution of Trp residues to the total near-UV fluorescence of NIR FPs.

\begin{tabular}{|c|c|c|c|}
\hline & iRFP713/iRFP682 variants & BphP1-FP variants & iRFP670 variants \\
\hline The maximum of total fluorescence $(\mathrm{nm})$ & 333 & 330 & 328 \\
\hline Fluorescence of $\operatorname{Trp} 95^{\mathrm{a}}$ & & & Not assigned \\
\hline Fluorescence of $\operatorname{Trp} 108^{a}$ & Quenched & & \\
\hline Fluorescence of Trp281 & $\begin{array}{l}\text { Blue-shifted } \\
\text { (The Trp has identical envir } \\
\text { proteins) }\end{array}$ & onment in both & $\begin{array}{l}\text { The most blue-shifted } \\
\text { (The Trp has the most hydrophobic environment among studied NIR FPs) }\end{array}$ \\
\hline Fluorescence of $\operatorname{Trp} 311^{\mathrm{a}}$ & Slightly red-shifted & & \\
\hline
\end{tabular}

\footnotetext{
${ }^{a}$ The cell filled with grey if NIR FP and its variants do not contain the Trp residue.
} 


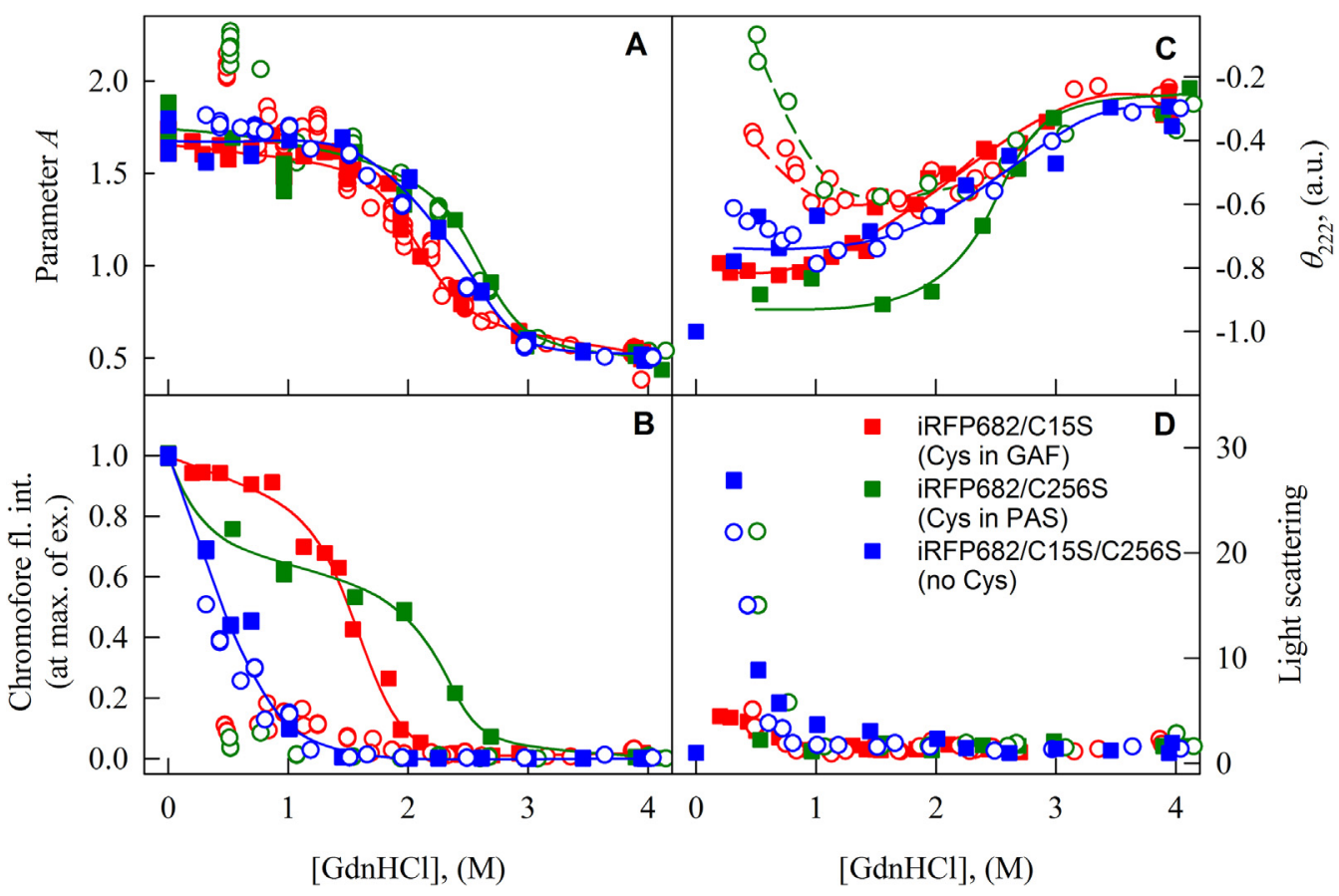

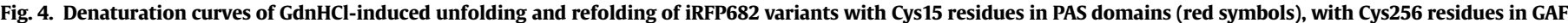

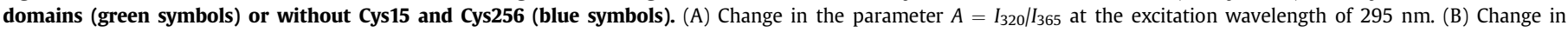

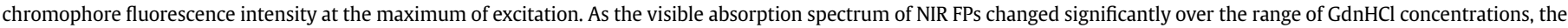

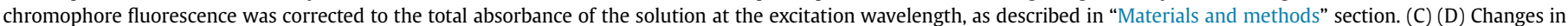

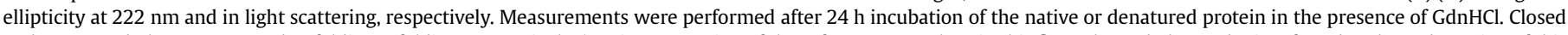

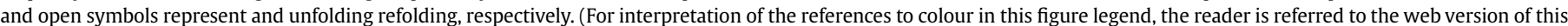
article.)

absorption band (Fig. 4 and Fig. S13). The dimeric NIR FPs, with Cys residues in both PAS and GAF domains, were able to bind BV covalently in both monomers [22]. This was explained by allosterical influence of the structural changes induced by Cys 15 in PAS domain to the structure of GAF domains. Data presented in current study confirmed that NIR FPs with Cys residues in PAS and GAF domains indeed had the most compact and stable structure. Higher stability of NIR FP variants with Cys15 in the PAS domains compared to NIR FP variants with Cys256 in the GAF domains may also be caused by structural changes introduced by Cys15. The structure of NIR FP variants with Cys15 in the PAS domains may be additionally fastened by the threading of BV-containing N-terminal part of the protein through the knot. The importance of the knot for interaction with BV is indicated by tolerance of iRFP713 to a circular permutation mainly at N-terminal tail of PAS domain and at unstructured loop between PAS and GAF domains, but not at knotforming loop of GAF-domain [54].

In the case of iRFP670 variants, the mutant form containing residues Cys15 and Cys256, possessed the highest stability similarly to corresponding mutant forms of the other NIR FPs. The other mutant variants of iRFP670 had substantially lower stability comparable to that of apoprotein. Remarkably, the stability of iRFP670 exceeded the stability of all other NIR FPs with Cys15 and Cys256, the chromophore dissociation in iRFP670 occurred at higher denaturant concentration (Fig. S13). At the same time, the denaturation of iRFP670, and of BphP1-FP, was less cooperative then the transition of iRFP682 and iRFP713 variants with Cys15 and Cys256. Contrary, iRFP670/C256S, bearing Cys15 in PAS domains, showed the lower stability and weaker chromophore association among NIR FPs with Cys15 in PAS domains (Fig. S13). NIR FPs with Cys256 in GAF domains had closer stability but the strength of the chromophore association was maximal for BphP1-FP/C15S (Fig. S13) compared to other NIR FPs with Cys256 in GAF domains. Similar behavior was observed for NIR FPs without Cys15 and Cys256. In spite of close stability of their structure, NIR FPs without Cys15 and Cys256 differed in the strength of the chromophore association with the protein. The chromophore was more tightly bound with BphP1-FP/C15S/C256I and more weakly with iRFP670/C15S/C256S (Fig. S13). These findings suggest the difference of the chromophore interaction with protein microenvironment in mutant forms iRFP670, BphP1-FP and iRFP682/iRFP713.

\section{Conclusion}

We studied the structural and spectral characteristics of NIR FPs, engineered from different bacterial phytochromes. The experiments on unfolding and refolding of NIR FPs, with a different location of cysteine residues capable of covalent BV binding, revealed that the change of the position of covalent attachment of the chromophore in NIR FPs did not recover their refolding. Intermolecular contacts were important for refolding of dimeric NIR FPs. NIR FPs with the chromophore incorporated into the GAF domain but unbound covalently had the less compact and stable protein structure. The formation of covalent bond between the chromophore and residue Cys15 or Cys256 stabilizes the structure of the NIR FPs to different extent. The higher stabilization of protein in the case of biliverdin attachment to Cys 15 is probably determined by a figure-of-eight knot. Indeed, passing of $\mathrm{N}$-terminal region through the knot with subsequent covalent bonding of this path and BV in the pocket of the GAF domain can make something like a pin. NIR FPs with both residues Cys15 and Cys256 exhibited the highest stability among all NIR FPs, despite the covalent linkage of the chromophore to the Cys256 in these proteins [22]. We believe that significant impact on the stability of NIR FPs with both residues 


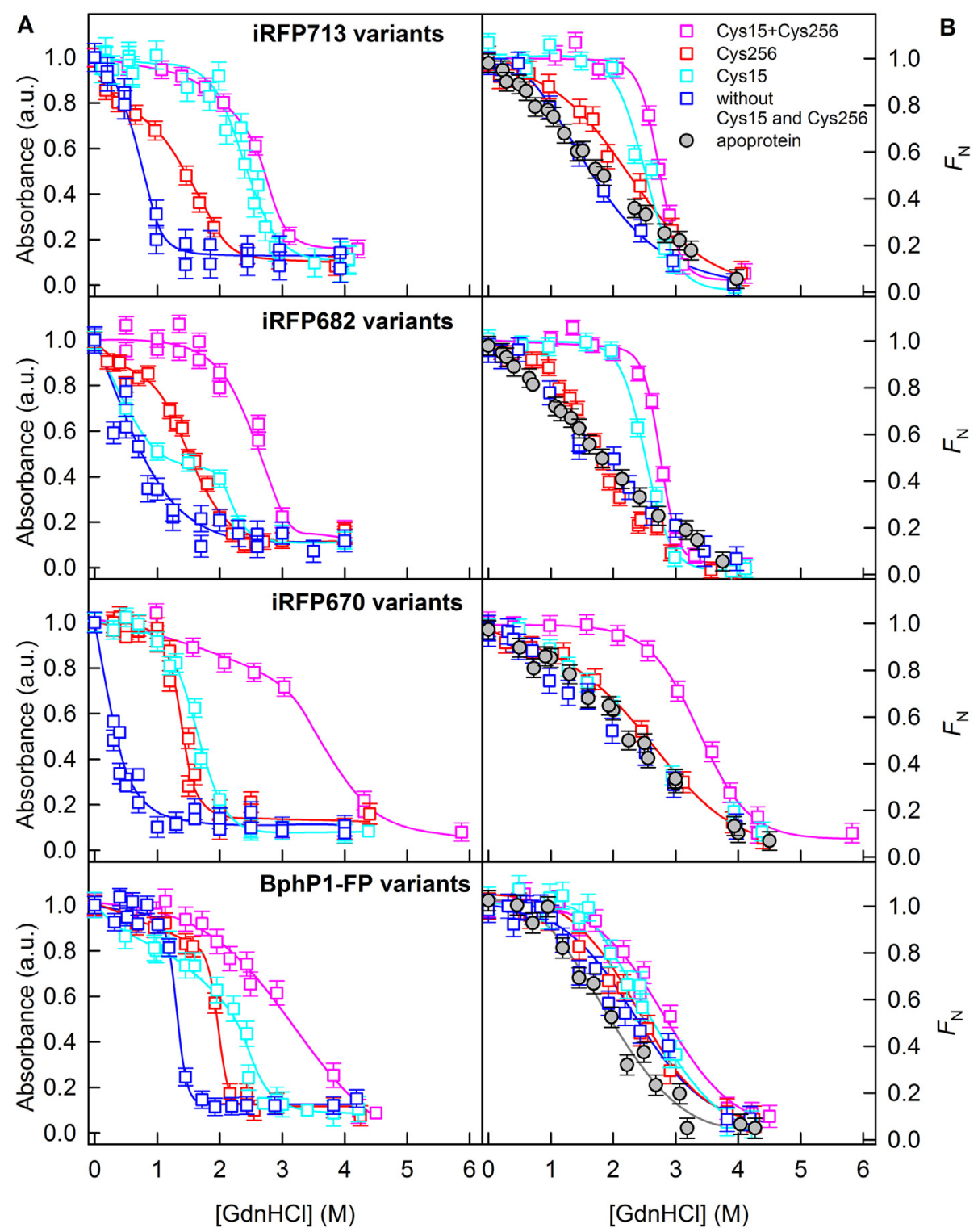

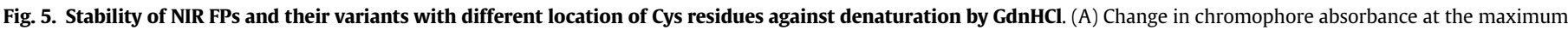
of absorption peak at the visible region. (B) Dependences of the part of native molecules $F_{\mathrm{N}}$ on $\mathrm{GdnHCl}$ concentration calculated on the basis of ellipticity at 222 nm.

Cys15 and Cys256 is provided by the structural changes introduced by cysteine in PAS domains.

\section{Acknowledgements}

This work was supported by the Russian Foundation for Basic Research (grant 16-04-01515 to O.V.S.), the Molecular and Cell Biology Program of the Russian Academy of Sciences (to K.K.T.) and the Government of Saint-Petersburg (grant 34 to O.V.S.).

\section{Appendix A. Supplementary data}

Supplementary data related to this article can be found at http:// dx.doi.org/10.1016/j.molstruc.2016.10.095.

\section{References}

[1] R.Y. Tsien, Integr. Biol. (Camb) 2 (2010) 77-93, http://dx.doi.org/10.1039/ b926500g.
[2] E. Betzig, Angew. Chem. Int. Ed. Engl. 54 (2015) 8034-8053, http://dx.doi.org/ 10.1002/anie.201501003.

[3] O.V. Stepanenko, O.V. Stepanenko, D.M. Shcherbakova, I.M. Kuznetsova, K.K. Turoverov, V.V. Verkhusha, Biotechniques 51 (2011) 313-327, http:// dx.doi.org/10.2144/000113765.

[4] D.M. Shcherbakova, M. Baloban, V.V. Verkhusha, Curr. Opin. Chem. Biol. 27 (2015) 52-63, http://dx.doi.org/10.1016/j.cbpa.2015.06.005.

[5] S.H. Bhoo, S.J. Davis, J. Walker, B. Karniol, R.D. Vierstra, Nature 414 (2001) 776-779, http://dx.doi.org/10.1038/414776a.

[6] G.S. Filonov, V.V. Verkhusha, Chem. Biol. 20 (2013) 1078-1086, http:// dx.doi.org/10.1016/j.chembiol.2013.06.009.

[7] K.D. Piatkevich, F.V. Subach, V.V. Verkhusha, Chem. Soc. Rev. 42 (2013) 3441-3452, http://dx.doi.org/10.1039/c3cs35458j.

[8] K.D. Piatkevich, F.V. Subach, V.V. Verkhusha, Nat. Commun. 4 (2013) 2153, http://dx.doi.org/10.1038/ncomms3153.

[9] G.S. Filonov, K.D. Piatkevich, L.M. Ting, J. Zhang, K. Kim, V.V. Verkhusha, Nat. Biotechnol. 29 (2011) 757-761, http://dx.doi.org/10.1038/nbt.1918.

[10] X. Shu, A. Royant, M.Z. Lin, T.A. Aguilera, V. Lev-Ram, P.A. Steinbach, R.Y. Tsien, Science 324 (2009) 804-807, http://dx.doi.org/10.1126/science.1168683.

[11] M.E. Auldridge, K.A. Satyshur, D.M. Anstrom, K.T. Forest, J. Biol. Chem. 287 (2012) 7000-7009, http://dx.doi.org/10.1074/jbc.M111.295121.

[12] M. Chen, W. Li, Z. Zhang, S. Liu, X. Zhang, X.E. Zhang, Z. Cui, Biomaterials 48 (2015) 97-107, http://dx.doi.org/10.1016/j.biomaterials.2015.01.038.

[13] K. Moffat, Philos. Trans. R. Soc. Lond. B Biol. Sci. 369 (2014) 20130568, http:// 
dx.doi.org/10.1098/rstb.2013.0568.

[14] D. Buhrke, F. Velazquez Escobar, L. Sauthof, S. Wilkening, N. Herder, N.N. Tavraz, M. Willoweit, A. Keidel, T. Utesch, M.A. Mroginski, F.J. Schmitt, P. Hildebrandt, T. Friedrich, Sci. Rep. 6 (2016) 28444, http://dx.doi.org/ $10.1038 /$ srep28444.

[15] T. Lamparter, M. Carrascal, N. Michael, E. Martinez, G. Rottwinkel, J. Abian, Biochemistry 43 (2004) 3659-3669, http://dx.doi.org/10.1021/bi0356931.

[16] T. Lamparter, N. Michael, O. Caspani, T. Miyata, K. Shirai, K. Inomata, J. Biol. Chem. 278 (2003) 33786-33792, http://dx doi org/10.1074/jbc.M305563200.

[17] D.M. Shcherbakova, M. Baloban, S. Pletnev, V.N. Malashkevich, H. Xiao, Z. Dauter, V.V. Verkhusha, Chem. Biol. 22 (2015) 1540-1551, http:// dx.doi.org/10.1016/j.chembiol.2015.10.007.

[18] K.A. Rumyantsev, D.M. Shcherbakova, N.I. Zakharova, A.V. Emelyanov, K.K. Turoverov, V.V. Verkhusha, Sci. Rep. 5 (2016) 18348, http://dx.doi.org/ $10.1038 /$ srep 18348 .

[19] J.R. Wagner, J. Zhang, J.S. Brunzelle, R.D. Vierstra, K.T. Forest, J. Biol. Chem. 282 (2007) 12298-12309, http://dx.doi.org/10.1074/jbc.M611824200.

[20] J.I. Sulkowska, E.J. Rawdon, K.C. Millett, J.N. Onuchic, A. Stasiak, Proc. Natl. Acad. Sci. U. S. A. 109 (2012) E1715-E1723, http://dx.doi.org/10.1073/ pnas.1205918109.

[21] J.R. Wagner, J.S. Brunzelle, K.T. Forest, R.D. Vierstra, Nature 438 (2005) 325-331, http://dx.doi.org/10.1038/nature04118.

[22] O.V. Stepanenko, M. Baloban, G.S. Bublikov, D.M. Shcherbakova, O.V. Stepanenko, K.K. Turoverov, I.M. Kuznetsova, V.V. Verkhusha, Sci. Rep. 6 (2016) 18750, http://dx.doi.org/10.1038/srep18750.

[23] D.M. Shcherbakova, V.V. Verkhusha, Nat. Methods 10 (2013) 751-754, http:/ dx.doi.org/10.1038/nmeth.2521.

[24] U.K. Laemmli, Nature 227 (1970) 680-685, http://dx.doi.org/10.1038/ $227680 \mathrm{a} 0$.

[25] O.V. Stepanenko, I.M. Kuznetsova, K.K. Turoverov, C. Huang, C.C. Wang Biochemistry 43 (2004) 5296-5303, http://dx.doi.org/10.1021/bi0359325.

[26] K.K. Turoverov, I.M. Kuznetsova, J. Fluoresc. 13 (2003) 41-57, http:// dx.doi.org/10.1021/bi00757a015.

[27] K.K. Turoverov, A.G. Biktashev, A.V. Dorofeiuk, I.M. Kuznetsova, Tsitologiia 40 (1998) 806-817.

[28] N.C. Rockwell, J.C. Lagarias, Chemphyschem 11 (2010) 1172-1180, http:// dx.doi.org/10.1002/cphc.200900894.

[29] D. Bellini, M.Z. Papiz, Acta Crystallogr. D. Biol. Crystallogr. 68 (2012) 1058-1066, http://dx.doi.org/10.1107/S0907444912020537.

[30] S. Dutta, K. Burkhardt, J. Young, G.J. Swaminathan, T. Matsuura, K. Henrick, H. Nakamura, H.M. Berman, Mol. Biotechnol. 42 (2009) 1-13, http:/ dx.doi.org/10.1007/s12033-008-9127-7.

[31] I.M. Kuznetsova, O.V. Stepanenko, K.K. Turoverov, M. Staiano, V. Scognamiglio, M. Rossi, S. D'Auria, J. Proteome Res. 4 (2005) 417-423, http://dx.doi.org/ 10.1021/pr0498077.

[32] I.M. Kuznetsova, T.A. Yakusheva, K.K. Turoverov, FEBS Lett. 452 (1999)
205-210, http://dx.doi.org/10.1016/S0014-5793(99)00574-8.

[33] K.K. Turoverov, I.M. Kuznetsova, V.N. Zaitsev, Biophys. Chem. 23 (1985) 79-89, http://dx.doi.org/10.1016/0301-4622(85)80066-1.

[34] A. Giordano, C. Russo, C.A. Raia, I.M. Kuznetsova, O.V. Stepanenko, K.K. Turoverov, J. Proteome Res. 3 (2004) 613-620, http://dx.doi.org/10.1021/ pr034132d.

[35] T. Förster, Rad. Res. Suppl. 2 (1960) 326, http://dx.doi.org/10.2307/3583604.

[36] R.E. Dale, J. Eisinger, Biopolymers 13 (1974) 1573, http://dx.doi.org/10.1002/ bip.1974.360130807.

[37] Y. Yamamoto, J. Tanaka, Bull. Chem. Soc. Jpn. 5 (1972) 1362, http://dx.doi.org/ 10.1246/bcsj.45.1362.

[38] T. Yamane, T. Andou, T. Ashida, Acta Cryst. B 33 (1977) 1650, http://dx.doi.org/ $10.1107 /$ S0567740877006815.

[39] Q. Chae, P.S. Song, J. Am. Chem. Soc. 97 (1975) 4176-4183, http://dx.doi.org/ $10.1021 / \mathrm{ja00848a004.}$

[40] T.W. Förster, Delocalized excitation and excitation transfer, in: Modern Quantum Chemistry, Academic Press, New York, 1965.

[41] J. Eisinger, B. Feuer, A.A. Lamola, Biochemistry 8 (1969) 3908-3915, http:// dx.doi.org/10.1021/bi00838a005.

[42] F. Kleima, E. Hofmann, B. Gobets, I.H. van Stokkum, R. van Grondelle, K. Diederichs, H. van Amerongen, Biophys. J. 78 (2000) 344, http://dx.doi.org/ 10.1016/S0006-3495(00)76597-0.

[43] A.F. McDonagh, L.A. Palma, Biochem. J. 189 (1980) 193-208.

[44] O.V. Stepanenko, G.S. Bublikov, D.M. Shcherbakova, V.V. Verkhusha, K.K. Turoverov, I.M. Kuznetsova, FEBS J. 281 (2014) 2284-2298, http:// dx.doi.org/10.1111/febs.12781.

[45] I.M. Kuznetsova, A.I. Sulatskaya, O.I. Povarova, K.K. Turoverov, PLoS One 7 (2012) e40845, http://dx.doi.org/10.1371/journal.pone.0040845.

[46] A.V. Fonin, A.I. Sulatskaya, I.M. Kuznetsova, K.K. Turoverov, PLoS One 9 (2014) e103878, http://dx.doi.org/10.1371/journal.pone.0103878.

[47] B. Nolting, Protein Folding Kinetics. In Biophysical Methods, Springer-Verlag Berlin-Heidelberg, 1999.

[48] S.W. Provencher, J. Glockner, Biochemistry 20 (1981) 33-37, http:// dx.doi.org/10.1021/bi00504a006.

[49] E.A. Burstein, Luminescence of Protein Chromophores (Model Investigations), vol. 6, VINITI, Moscow, 1976.

[50] J.N. Scott, P.R. Callis, J. Phys. Chem. B 117 (2013) 9598-9605, http://dx.doi.org/ 10.1021/jp4041716.

[51] P.R. Callis, J. Mol. Struct. 1077 (2014) 22-29, http://dx.doi.org/10.1016/ j.molstruc.2014.04.051.

[52] R.M. Hochstrasser, D.K. Negus, Proc. Natl. Acad. Sci. U. S. A. 81 (1984) 4399-4403.

[53] I.M. Kuznetsova, K.K. Turoverov, Tsitologiia 40 (1998) 747-762.

[54] N. Pandey, B.E. Kuypers, B. Nassif, E.E. Thomas, R.N. Alnahhas, L. Segatori, J.J. Silberg, Biochemistry 55 (2016) 3763-3773, http://dx.doi.org/10.1021/ acs.biochem.6b00258. 